\title{
Pacific
}

Journal of

Mathematics

\section{ORIENTED ORBIFOLD COBORDISM}

KIMBERLy Sue DRUSCHEL 


\title{
ORIENTED ORBIFOLD COBORDISM
}

\author{
K. S. DRUSChEL
}

A complete set of invariants (generalized Pontrjagin numbers) for rational oriented orbifold cobordism is determined. Using these numbers we prove that for any odd dimensional oriented orbifold $Q$ there is a nonzero multiple of $Q$ which bounds another orbifold and that, unlike the manifold case, this need not be true for $4 k+2$ dimensional orbifolds. In addition we construct generators for the rational orbifold cobordism ring and show that it is a free commutative ring on these.

Introduction. This paper establishes a foundation for oriented orbifold cobordism in a manner analogous to Thom's results for oriented manifolds. Thom's main theorem states that some multiple of an oriented compact manifold bounds another such manifold if and only if its Pontrjagin numbers are zero. Orbifolds are like manifolds, except that they locally look like $\mathbf{R}^{n} / G$ for $G$ a finite group. For each $H$ a finite subgroup of $\mathrm{SO}(n)$ we define $H$ characteristic numbers of the compact oriented orbifold $Q$ which account for its $H$ singular set and are its Pontrjagin numbers when $H$ is trivial. Our main theorem is that some multiple of $Q$ bounds another compact oriented orbifold if and only if all its $H$ characteristic numbers are zero for all finite subgroups $H$ of $\mathrm{SO}(n)$.

Thom's corollary that every manifold with dimension not divisible by four rationally bounds does not translate exactly in the orbifold case. There is no reason to expect it to as the $H$ singular set of even an odd dimensional orbifold may have nonzero Pontrjagin numbers. However, a careful consideration of orientations and the twisted cohomology of the classifying space of the centralizer of $H, C_{\mathrm{O}(d)}(H)$, shows that any odd dimensional orbifold rationally bounds another orbifold $W$ with the same set of local groups. The result for $(4 k+2)$ dimensional orbifolds is not as neat. We can isolate a certain class of finite subgroups of $\mathrm{SO}(4 k+2)$, which includes all finite abelian subgroups, such that any $(4 k+2)$-dimensional orbifold with all local groups in this class rationally bounds. This set does not include all finite subgroups as we construct a seventy-dimensional orbifold with nonzero characteristic numbers which therefore does not rationally bound. 
The article begins with a preliminary section on orbifolds, orbibundles, orientations, and orbifold cobordism. The second section is devoted to defining the $H$ characteristic numbers and proving that they rationally determine orbifold cobordism. The proof adopts the local techniques of Lee and Wasserman [8]. We follow this with an analysis of these numbers and the cohomology from which they arise, yielding the odd and $(4 k+2)$-dimensional results. Using the work of the previous two sections, in the fourth section we construct orbifolds which form bases for the rational orbifold cobordism groups and show that $\Omega_{*, \text { orb }} \otimes \mathbf{Q}$ is a free commutative algebra over $\Omega_{*} \otimes \mathbf{Q}$ with generators a certain subset of these orbifolds.

This article stems from my dissertation, which was directed by Professor Michael Davis, to whom I am very grateful for his help and suggestions. I would also like to thank Professor Walter Neumann for his suggestion concerning the $(4 k+2)$-dimensional counterexample.

\section{Preliminaries.}

DEFINITION 1.1 . Let $X$ be a topological space.

(i) An orbifold chart on $X$ is a four-tuple $(U, G, \bar{U}, \pi)$ with $U$ an open subset of $X, \bar{U}$ an open subset of $\mathbf{R}^{n}, G$ a finite group of diffeomorphisms acting effectively on $\bar{U}$, and $\pi: \bar{U} \rightarrow U$ an open map onto $U$ such that $\pi=h \pi_{U}$ where $\pi_{U}$ is the natural orbit map and $h$ is a homeomorphism from $\bar{U} / G$ to $U$.

(ii) Two charts $\left(U_{i}, G_{i}, \bar{U}_{i}, \pi_{i}\right), i=1,2$, on $X$ are compatible if for every $x \in U_{1} \cap U_{2}$ there is an open set $U \subset \bar{U}_{1}$ with a lift $\bar{x}$ of $x$ contained in $U$ and a diffeomorphism $f: U \rightarrow \bar{U}_{2}$, called an overlap map, such that $\pi_{2} f=\pi_{1}$.

(iii) An orbifold atlas $\mathscr{U}$ on $X$ is a collection $\left\{\left(U_{\alpha}, G_{\alpha}, \bar{U}_{\alpha}, \pi_{\alpha}\right)\right\}_{\alpha \in \Lambda}$ of compatible charts on $X$ such that $\bigcup_{\alpha \in \Lambda} U_{\alpha}=X$.

Definition 1.2. A smooth orbifold $Q$ is a Hausdorff space $|Q|$, called the underlying space, together with a maximal orbifold atlas $\mathscr{U}$ on $|Q|$.

Definition 1.3. An orbifold with boundary $W$ is defined by allowing $\bar{U}$ to be an open subset of $H^{n}=\left\{\left(x_{1}, \ldots, x_{n}\right) \in \mathbf{R}^{n}: x_{n} \geq 0\right\}$ in the above definition. The orbifold boundary of $W$, denoted $\partial_{o} W$, has underlying space $\left|\partial_{o} W\right|=\left\{w \in|W|: w \in \pi\left(\partial\left(\bar{U} \cap H^{n}\right)\right)\right.$ for some chart of $W\}$ and the obvious orbifold structure.

An orbifold diffeomorphism is defined by a homeomorphism $f:|Q|$ $\rightarrow\left|Q^{\prime}\right|$ such that $(U, G, \bar{U}, \pi)$ is in the maximal atlas for $Q$ if and only if $(f(U), G, \bar{U}, f \pi)$ is a chart in the maximal atlas of $Q^{\prime}$. 
Definition 1.4. Let $x \in|Q|$ and $(U, G, \bar{U}, \pi)$ be a chart for $Q$ with $x \in U$ and $y \in \bar{U}$ such that $\pi(y)=x$. The local group at $x$ is defined to be $G_{y}$, the isotropy group of $y$.

We have that $G_{y}$ is isomorphic to the linear group formed by its differentials at $y$. If $\left(U_{i}, G_{i}, \bar{U}_{i}, \pi_{i}\right)$ are two charts with $x \in U_{i}$ and $\pi_{i}\left(y_{i}\right)=x$ and $h$ is an overlap map sending $y_{1}$ to $y_{2}$ then conjugation by $d h_{y_{1}}$ provides an isomorphism between $d G_{y_{1}}$ and $d G_{y_{2}}$. It follows that the local group at $x$ is independent of the choice of chart and is well defined up to conjugacy as a subgroup of $\operatorname{Gl}(n, \mathbf{R})$ where $n=\operatorname{dim} Q$.

Definition 1.5. The set of conjugacy classes in $\operatorname{Gl}(n, \mathbf{R})$ of local groups of $Q$ is denoted by $\mathscr{G}_{Q}$ and the class of a subgroup $H$ in $\mathscr{G}_{Q}$ is denoted by $(H)$. The set $\mathscr{G}_{Q}$ is partially ordered by $(H) \leq\left(H^{\prime}\right)$ if $H$ is conjugate in $\operatorname{Gl}(n, \mathbf{R})$ to a subgroup of $H^{\prime}$. Note that $\mathscr{G}_{Q}$ is finite if $Q$ is compact.

Many of the concepts one would want in a theory of orbifolds are most precisely defined in the language of groupoids. This approach can be found in [7] and in more detail in [5].

Definition 1.6. For $\mathscr{U}=\left\{\left(U_{\alpha}, G_{\alpha}, \bar{U}_{\alpha}, \pi_{\alpha}\right)\right\}_{\alpha \in \Lambda}$, an atlas for $Q$, there is the topological groupoid $\Gamma_{\mathscr{U}}$ whose set of objects is $\widetilde{Q} \stackrel{\text { def }}{=}$ $\left\{x: x \in \amalg_{\alpha \in \Lambda} \bar{U}_{\alpha}\right\}$ and whose morphisms are the germs of local diffeomorphisms which commute with the natural map $\pi$ from $\tilde{Q}$ to $|Q|$. Thus the morphisms are generated by the germs of local groups and overlap maps. $\Gamma_{\mathscr{U}}$ has the "germ" topology $([5,3.7])$.

Definition 1.7. $Q$ is orientable if there is an orientation of $\widetilde{Q}$ which is preserved by $\Gamma_{\mathscr{U}}$. A weaker notion is that of local orientability which means that all the local groups are subgroups of $\mathrm{Gl}(n, \mathbf{R})_{+}$.

Suppose $F$ is a topological space and $G$ is a topological group acting effectively on $F$. As a generalization of $(F, G)$ bundles we have:

Definition 1.8. An $(F, G)$ orbibundle $E \stackrel{p}{\rightarrow} Q$ is defined as an $(F, G)$ bundle $\widetilde{E} \stackrel{\tilde{p}}{\rightarrow} \widetilde{Q}$ with a $\Gamma_{\mathscr{U}}$ action (see [7, §2.2]) on $\widetilde{E}$ through $(F, G)$ bundle isomorphisms which extends the natural action on $\widetilde{Q}$.

There is an induced map $\widetilde{E} / \Gamma_{\mathscr{U}} \stackrel{p}{\rightarrow}|Q|$ of $\Gamma_{\mathscr{U}}$ quotients and $p^{-1}\left(U_{\alpha}\right) \cong \bar{U}_{\alpha} \times_{G_{\alpha}} F$ where $G_{\alpha}$ acts on $F$ by elements of $G$. If $F$ is an orbifold, $G<\operatorname{Diffeo}(F), \widetilde{E}$ is a smooth $(F, G)$ bundle and 
$\Gamma_{\mathscr{U}}$ acts smoothly on $\widetilde{E}$, then $\widetilde{E} / \Gamma_{\mathscr{U}}$ has a natural orbifold structure which we often denote by $E$.

Many of the usual notions for bundles carry through to orbibundles, including associated principal orbibundles and reduction of the structure group $G,[13, \S 1]$. Vector bundles are now generalized to $\left(\mathbf{R}^{n}, \mathrm{Gl}(n, \mathbf{R})\right)$-orbibundles, called orbivector bundles. Riemannian metrics on orbivector bundles are reductions of their structure group to $\mathrm{O}(n)$. An oriented orbibundle would be one where $\widetilde{E}$ is an oriented $(F, G)$ bundle and $\Gamma_{\mathscr{U}}$ preserves the orientation.

The most revelant examples for this paper include the tangent bundle to $Q, T Q$, (which is $T \widetilde{Q}$ with $\Gamma_{\mathscr{U}}$ acting by differentials), its associated principal $\mathrm{Gl}(n, \mathbf{R})$ orbibundle, $P Q$, and, given a Riemannian metric $g$ on $Q$, the associated principal $\mathrm{O}(n)$ orbibundle, $P_{g} Q$. We note that $P Q$, respectively $P_{g} Q$, are smooth manifolds and that there is a smooth right $\mathrm{Gl}(n, \mathbf{R})$, respectively $\mathrm{O}(n)$, action with finite isotropy groups whose orbit space is $Q$. Thus since any paracompact orbifold admits a Riemannian metric, we conclude that any compact orbifold is the quotient of a compact manifold (of higher dimension) by a smooth compact Lie group action with finite isotropy groups.

For the groupoid $\Gamma_{\mathscr{U}}$ there is a universal principal $\Gamma_{\mathscr{U}}$ bundle [7, $\S 3.1] E \Gamma_{\mathscr{U}} \stackrel{\tilde{\pi}}{\rightarrow} B \Gamma_{\mathscr{U}}$ and natural maps $\psi_{\mathscr{U}}: B \Gamma_{\mathscr{U}} \rightarrow|Q|, \hat{\psi}_{\mathscr{U}}: E \Gamma_{\mathscr{U}} \rightarrow$ $\widetilde{Q}$ making the appropriate diagram commutative. If $\mathscr{U}^{\prime}$ is another atlas for $Q, B \Gamma_{\mathscr{U}^{\prime}}$ is homotopy equivalent to $B \Gamma_{\mathscr{U}}$ by a homotopy $h$ such that $h \psi_{\mathscr{U}}=\psi_{\mathscr{U}}$ [5, Remark 8.1]. Thus we often denote $B \Gamma_{\mathscr{U}}$ by $B Q$ and $\psi_{\mathscr{U}}$ by $\psi$. One model for $B Q$ is $P_{g} Q \times_{\mathrm{O}(n)} E \mathrm{O}(n)$ ([5, Remark 8.2]). Also a map of orbifolds $\lambda: Q^{\prime} \rightarrow Q$ is defined by a generalized homomorphism $[5, \S 4.8 ; 7, \S 2.3] \hat{\lambda}$ between $\Gamma_{\mathscr{U}^{\prime}}$ and $\Gamma_{\mathscr{U}}$ and so defines a homotopy class of maps $B \lambda: B Q^{\prime} \rightarrow B Q$. Here $\mathscr{U}^{\prime}$ and $\mathscr{U}$ are atlases for $Q^{\prime}$ and $Q$, respectively .

For $V$ an $(F, G)$ orbibundle over $Q$ we denote by $\psi^{*} V$ the bundle $\hat{\psi}_{\mathscr{U}}^{*} \widetilde{E} / \Gamma_{\mathscr{U}}$. Then since $\Gamma_{\mathscr{U}}$ acts freely on $E \Gamma_{\mathscr{U}}$ we have the following.

LEMMA 1.9. $\psi^{*} V$ is an ordinary $(F, G)$ bundle and so is classified by a map $f: B Q \rightarrow B G$.

If the structure group of the orbibundle $V$ reduces to $L<G$ then $f$ lifts to a classifying map to $B L$. Also for the orbifold map $\lambda: Q^{\prime} \rightarrow$ $Q, \lambda^{*} V$ is defined by the bundle $V \times_{\Gamma_{\mathscr{U}}} \hat{\lambda}$ over $\widetilde{Q}^{\prime}$ with $\Gamma_{\mathscr{U}^{\prime}}$ acting on the right of $\hat{\lambda}$. Working through the definitions one checks that 
$(B \lambda)^{*} \psi^{*} V=\psi^{\prime *} \lambda^{*} V$. Here $\psi^{\prime}: B Q^{\prime} \rightarrow\left|Q^{\prime}\right|$ is the canonical map.

From $[5,6.4]$ and $[7,4.2 .4]$ we have

LEMMA 1.10. If $Q$ is a locally orientable, $n$-dimensional orbifold then $|Q|$ is a rational homology manifold.

Thus as in [16, §2] $|Q|$ has a twisted fundamental class $[Q] \in$ $H_{n}(|Q| ; \widehat{\mathbf{Q}})$, where $\widehat{\mathbf{Q}}$, or or $_{Q}$, denotes the orientation system of $|Q|$. We also have that if $E \stackrel{p}{\rightarrow} Q$, denoted $\phi$, is a locally orientable $\left(\mathbf{R}^{n} / K, G\right)$-orbibundle with $K$ finite and orientation preserving and $G<N_{\mathrm{Gl}(n, \mathbf{R})}(K) / K$, where $N_{\mathrm{Gl}(n, \mathbf{R})}(K)$ is the normalizer of $K$ in $\mathrm{Gl}(n, \mathbf{R})$, then $H_{n}\left(\left|E_{x}\right|,\left|E_{x}-0\right| ; \mathbf{Q}\right), x \in|Q|$, defines an orientation system for $\phi$, denoted or $_{\phi}$ or $\widehat{\mathbf{Q}}$. In general, in $H^{n}\left(|E|,\left|E_{0}\right| ; p^{*}\right.$ or $\left._{\phi}\right)$ there is a unique class, $\mu_{\phi}$, called the Thom class of $\phi$ such that $\bigcup \mu_{\phi}: H^{k}(|E| ; \Gamma) \cong H^{n+k}\left(|E|,\left|E_{0}\right| ; \Gamma \otimes p^{*}\right.$ or $\left._{\phi}\right)$ for any rational local system $\Gamma$. If $e \in H^{n}\left(|Q| ;\right.$ or $\left._{\phi}\right)$ is the class which gets mapped onto $\mu_{\phi}$ by $p^{*}$ then $e$ is called the twisted Euler class of $\phi$. Also if $Q^{\prime}$ is the orientation double cover of $Q$ associated with $\phi$ then $H^{*}\left(|Q| ;\right.$ or $\left._{\phi}\right) \cong H^{*}\left(\left|Q^{\prime}\right| ; \mathbf{Q}\right)^{-}$, the -1 eigenvectors of $\alpha^{*}$ where $\alpha$ is the involution of the cover.

Lemma 1.11. Suppose $Q$ is a locally orientable orbifold. Then $\psi^{*}$ is a rational cohomology isomorphism, both for trivial coefficients and between $H^{*}\left(|Q|\right.$; or $\left._{Q}\right)$ and $H^{*}\left(B Q ; \psi^{*}\right.$ or $\left._{Q}\right)$.

Proof. The trivial coefficient case is proved in [5] and [7, 4.2.3]. One has that $H^{*}\left(B Q ; \psi^{*}\right.$ or $\left._{Q}\right)$ is isomorphic to the -1 eigenvectors of $B \alpha^{*}$ acting on $H^{*}\left(B Q^{\prime} ; \mathbf{Q}\right)$ and these coincide with $H^{*}\left(\left|Q^{\prime}\right| ; \mathbf{Q}\right)^{-}$ under the isomorphism $\psi^{\prime *}$ where $\psi^{\prime}: B Q^{\prime} \rightarrow\left|Q^{\prime}\right|$.

Assume that $\zeta$ is an orbivector bundle over a locally orientable orbifold $Q$ and that $V$ is a $\left(\mathbf{R}^{n} / K, G\right)$ orbibundle over $Q$ as above with $T Q \oplus V$ oriented.

Definition 1.12. (i) The $i$ th Pontrjagin class of $\zeta, p_{i}(\zeta)$, is defined to be $\left(\psi^{*}\right)^{-1}\left(p_{i}\left(\psi^{*}(\zeta)\right)\right)$.

(ii) Let $x \in H^{*}(B G$; $\widehat{\mathbf{Q}})$ where the coefficients are those of the canonical $\left(\mathbf{R}^{n} / K, G\right)$ bundle over $B G$; then $x(V) \in H^{*}\left(Q\right.$; or $\left._{Q}\right)$ is the class $\left(\psi^{*}\right)^{-1}\left(f^{*} x\right)$.

From the discussion following Lemma 1.9 we conclude 
LEMMA 1.13. Pontrjagin classes of orbivector bundles have all the usual properties, as in [12]; in particular, they respect direct sums, are zero for trivial bundles and natural with respect to orbifold maps. The other characteristic classes of orbibundles in the above definition have the latter two properties.

Definition 1.14. Suppose $|X| \subset|Q|$. We say that $X$ is a $k$-dimensional suborbifold of $Q$ if there is an atlas

$$
\mathscr{U}=\left\{\left(U_{\alpha}, G_{\alpha}, \bar{U}_{\alpha}, \pi_{\alpha}\right)\right\}_{\alpha \in \Lambda}
$$

for $Q$ such that for every $\alpha \in \Lambda$ with $U_{\alpha} \cap|X| \neq \varnothing, \bar{U}_{\alpha} \cap \mathbf{R}^{k}$ is invariant under $G_{\alpha}$, and $\pi_{\alpha}\left(\bar{U}_{\alpha} \cap \mathbf{R}^{k}\right)=U_{\alpha} \cap|X|$.

If $\Lambda^{\prime}=\left\{\alpha \in \Lambda: U_{\alpha} \cap|X| \neq \varnothing\right\}$ then $X$ has the orbifold structure given by the atlas $\mathscr{U}^{\prime}=\left\{\left(U_{\alpha} \cap|X|, G_{\alpha} / K_{\alpha}, \bar{U}_{\alpha} \cap \mathbf{R}^{k}, \pi_{\alpha \mid \bar{U}_{\alpha} \cap R^{k}}\right)\right\}_{\alpha \in \Lambda^{\prime}}$, where $K_{\alpha}$ is the (normal) subgroup of $G_{\alpha}$ which acts as the identity on $\bar{U}_{\alpha} \cap \mathbf{R}^{k}$.

Let $\bar{U}_{\alpha}^{\prime}$ denote $\bar{U}_{\alpha} \cap \mathbf{R}^{k}$. If $Q$ is a Riemannian orbifold then $T \bar{U}_{\alpha \mid \bar{U}_{\alpha}^{\prime}}$ decomposes as $T \bar{U}_{\alpha}^{\prime} \oplus \nu\left(\bar{U}_{\alpha}^{\prime}\right)$ and this is invariant under $\Gamma_{\mathscr{U}}$ restricted to $\coprod_{\alpha \in \Lambda^{\prime}} \bar{U}_{\alpha}^{\prime}$. Therefore $\coprod_{\alpha \in \Lambda^{\prime}} \nu\left(\bar{U}_{\alpha}^{\prime}\right) / K_{\alpha}$ defines a normal bundle $\nu(X)$ over $X .|X|$ can be written as $\amalg\left|X_{\beta}\right|$ where $\left(K_{\beta}\right)$ is constant for $x \in\left|X_{\beta}\right|$. Then $\nu\left(X_{\beta}\right)$ is a $\left(\mathbf{R}^{k} / K_{\beta}, N_{\mathrm{O}(k)}\left(K_{\beta}\right) / K_{\beta}\right)$ orbibundle.

From the equivariant tubular neighborhood theorem [2] applied to $P_{g} Q$ ([6, Proposition 2.1.2]) we obtain

Proposition 1.15 (orbifold tubular neighborhood theorem). Let $Q^{\prime}$ be a suborbifold of the paracompact orbifold $Q^{n}$. Then there is a neighborhood $U$ of $Q^{\prime}$ in $Q$ which is orbifold diffeomorphic to the total space of the normal bundle $\nu\left(Q^{\prime}\right)$ of $Q^{\prime}$.

Similarly one has an Orbifold Collaring Theorem which says that if $W$ is a paracompact orbifold then $\partial_{o} W \times[0,1]$ is orbifold diffeomorphic to some neighborhood of $\partial_{o} W$ in $W$. By using the Collaring Theorem and standard arguments from the manifold case $[4, \S 1.3]$ one obtains the geometric theorems necessary for the development of orbifold cobordism. Namely, one can glue two orbifolds together along orbifold diffeomorphic orbifold boundaries to form a new orbifold and also that one can "straighten the angle" as for manifolds.

Definition 1.16. Suppose $Q_{i}, i=1,2$, are two closed, oriented, $n$-dimensional orbifolds. We say that $Q_{1}$ is oriented orbifold cobor- 
dant to $Q_{2}$, written $Q_{1} \sim_{o} Q_{2}$, if there is an oriented orbifold $W$ such that $Q_{1} \amalg-Q_{2}=\partial_{o} W$ with the inherited orientation.

Analogous to manifold cobordism, oriented orbifold cobordism is an equivalence relation on the set of closed, oriented, $n$-dimensional orbifolds. We denote the class of $Q$ by $[Q]_{o}$ and the set of oriented orbifold cobordism classes of $n$-dimensional orbifolds by $\Omega_{n \text {, orb }}$. $\Omega_{* \text {, orb }}$ is a graded ring via disjoint union and the cartesian product.

2. $H$ characteristic numbers. We assume henceforth that $Q$ is a smooth, oriented, Riemannian $n$-dimensional orbifold and that $H$ is a finite subgroup of $\mathrm{SO}(n)$ of degree $d$ (Definition 3.1).

We motivate our definition of $H$ characteristic numbers by first considering the case where $H$ is maximal in $\mathscr{G}_{Q}$. Then the $H$ singular set, $Q^{H}$ (see below), is a manifold. It follows from a twisted bordism theory that some multiple $m$ of the normal bundle $\nu_{H}$ of $Q^{H}$ bounds another bundle $E$ if and only if $\left\langle p_{I}\left(T Q^{H}\right) \cup f^{*} x,\left[Q^{H}\right]\right\rangle=0$ for all $x \in H^{*}\left(B N_{\mathrm{O}(d)}(H) / H ; \widehat{\mathbf{Q}}\right)$ and $p_{I}$ a product of Pontrjagin classes with $4|I|+\operatorname{dim} x=\operatorname{dim} Q^{H}$ (Lemma 2.8). Here $f$ is the classifying map of $\nu_{H}$. A standard geometric construction using $E$ then gives a cobordism between $m Q$ and an orbifold $Q^{\prime}$ with $\mathscr{G}_{Q^{\prime}} \subset \mathscr{G}_{Q}-(H)$ (Lemma 2.2).

For $H$ nonmaximal our $H$ characteristic numbers are orbifold characteristic numbers of the same form as above. To define these we construct an orbifold $Q_{H}$ and its normal bundle $\nu_{H}$ which are the best orbifold approximation of the $H$ singular set and a neighborhood of it in $Q$. As these numbers are orbifold cobordism invariants (Lemma 2.14), if all of them are 0 for all $H$ we can successively apply the above cobordism to make $Q$ rationally cobordant to a manifold whose Pontrjagin numbers are all zero and which thus rationally bounds. It then follows that some multiple of $Q$ bounds.

Definition 2.1. The $H$ singular set of $Q$ is $Q^{H} \stackrel{\text { def }}{=}\{x \in Q \mid(H)<$ $\left.\left(G_{x}\right)\right\}$.

Now suppose that $H$ is maximal in $\mathscr{G}_{Q}$. Then for a chart $(U, H$, $\bar{U}, \pi)$ for $Q$ with $U \cap Q^{H} \neq \varnothing$ we have $U \cap Q^{H}=\pi\left(\bar{U}^{H}\right)$ so $Q^{H}$ is a manifold and a suborbifold. Also for $\phi: U \subset \bar{U}_{1} \rightarrow \bar{U}_{2}$ an overlap map, $\left.d \phi\right|_{U^{H}}$ is in $N_{\mathrm{O}(n)}(H)$ and hence the normal bundle of $Q^{H}$, $\nu_{H}$, is an $\left(\mathbf{R}^{d} / H, N_{\mathrm{O}(d)}(H) / H\right)$-bundle.

Lemma 2.2. Suppose that $Q$ is a closed orbifold and that $\nu_{H}$ bounds. That is, there is a compact manifold $Z$ and a smooth 
$\left(\mathbf{R}^{d} / H, N_{\mathrm{O}(d)}(H) / H\right)$-bundle $\xi$ over $Z$ such that (i) $\partial Z=Q^{H}$, (ii) $\left.\xi\right|_{Q^{H}}=\nu_{H}$, and (iii) the orientation of the bundle $\left.(T Z \oplus \xi)\right|_{Q^{H}}$ is the same as that of $\nu\left(Q^{H}, Z\right) \oplus T Q^{H} \oplus \nu_{H}$. (Here the normal bundle of $Q^{H}$ in $Z, \nu\left(Q^{H}, Z\right)$ has outward direction positive.) Then $Q$ is oriented orbifold cobordant to an orbifold $Q^{\prime}$ with $\mathscr{G}_{Q^{\prime}} \subset \mathscr{G}_{Q}-(H)$ via an orbifold $W$ with $\mathscr{G}_{W}=\mathscr{G}_{Q}$.

Proof. Let $h: U \rightarrow \mathbf{D} \nu_{H}$ be an orientation preserving orbifold diffeomorphism from a closed tubular neighborhood $U$ of $Q^{H}$ onto the total space of $\mathbf{D} \nu_{H}$. (In general if $\eta$ is a $\left(\mathbf{R}^{d} / H, N_{\mathrm{O}(d)}(H) / H\right)$ orbibundle then $\mathbf{D} \eta$ and $\mathbf{S} \eta$ denote the associated orbibundles with fiber $\mathbf{D}^{d} / H$, respectively $\mathbf{S}^{d-1} / H$.) Let $W=(Q \times I \cup \mathbf{D} \xi) / \sim$ where $\sim$ is defined by identifying $(x, 1)$ in $U \times\{1\}$ with $h(x) \in \mathbf{D} \nu_{H} \subset \mathbf{D} \xi$. Once we straighten the angle at $\mathbf{S} \nu_{H}, W$ becomes an oriented orbifold with boundary $Q \amalg Q^{\prime}$, where $Q^{\prime}=(Q-(U-\partial U)) \times\{1\} \cup \mathbf{S} \xi / \sim^{\prime}$ with $\sim^{\prime}$ the above identification restricted to $\partial U \times\{1\}$. Since $\xi$ is an $\mathbf{R}^{d} / H$ bundle over a manifold, $\mathscr{G}_{\mathbf{D} \xi}=\mathscr{G}_{\mathbf{R}^{d} / H} \subset \mathscr{G}_{Q}$ and $\mathscr{G}_{\mathbf{S} \xi} \subset$ $\mathscr{G}_{\mathbf{R}^{d} / H}-(H)$ and hence $\mathscr{G}_{W}=\mathscr{G}_{Q}$ and $\mathscr{G}_{Q^{\prime}} \subset \mathscr{G}_{Q}-(H)$.

This leads us to consider the following twisted bordism theory. Suppose $\Upsilon$ is a $\left(\mathbf{R}^{d} / H, N_{\mathrm{O}(d)}(H) / H\right)$-bundle over a CW complex $B$ with finite skeleton in each dimension.

Definition 2.3. Consider pairs $\left(M^{l}, f\right)$, where $M^{l}$ is a compact manifold and $f: M \rightarrow B$ with $T M \oplus f^{*} \Upsilon$ oriented. We say that two such pairs $\left(M_{i}^{l}, f_{i}\right), i=1,2$, with $M_{i}^{l}$ closed, are twisted cobordant, denoted $\left(M_{1}, f_{1}\right) \sim_{t}\left(M_{2}, f_{2}\right)$, if there is a pair $\left(W^{l+1}, g\right)$ as above such that (i) $\partial W=M_{1} \amalg M_{2}$, (ii) $\left.g\right|_{M_{i}}=f_{i}$, and (iii) $\left.\left(T W \oplus g^{*} \Upsilon\right)\right|_{M_{1}}$ has the same orientation as $\nu\left(M_{1}\right) \oplus T M_{1} \oplus f_{1}^{*} \Upsilon$ and $\left.\left(T W \oplus g^{*} \Upsilon\right)\right|_{M_{2}}$ opposite the orientation of $\nu\left(M_{2}\right) \oplus T M_{2} \oplus f_{2}^{*} \Upsilon$, where $\nu\left(M_{i}\right)$, the normal bundle of $M_{i}$ in $W$, has outward direction as positive.

Let $[M, f]_{t}$ be the class of $(M, f)$ and $\Omega_{l, t}(\Upsilon)$ the group of these cobordism classes. $\Omega_{*, t}(\Upsilon)$ is a left $\Omega_{*}$ module via $[N][M, f]_{t}=$ [N $\left.\times M, f \pi_{2}\right]_{t}$ where $\pi_{2}$ is projection onto the second factor and $\Omega_{*}$ denotes the oriented manifold cobordism ring.

LEMMA 2.4. $\Omega_{l, t}(\Upsilon) \otimes \mathbf{Q} \cong \bigoplus_{j=0}^{l} \Omega_{j} \otimes H_{l-j}(B ; \widehat{\mathbf{Q}})$.

Proof. When $\Upsilon$ is oriented $f^{*} \Upsilon$ has an induced orientation and hence $M$ is oriented. Thus $\Omega_{l, t}(\Upsilon)=\Omega_{l}(B)$ and the lemma is a result of $[4$, Theorem 14.2]. Otherwise one traces arguments checking 
orientations ([6, Lemma 4.2.3]) to obtain

$$
\Omega_{l, t}(\Upsilon) \cong \pi_{l+k}\left(\mathscr{M}\left(\tilde{\gamma}^{k} \times_{\mathbf{Z}_{2}} \widetilde{B}\right), \infty\right) \text { for } k \geq l+2,
$$

where (a) $\widetilde{B}$ is the orientation double cover for $\Upsilon$, and (b) $\mathbf{Z}_{2}$ acts by the diagonal using (a) and the orientation reversing action on $\tilde{\gamma}^{k}$. Here $\tilde{\gamma}^{k}$ denotes the canonical $k$-dimensional vector bundle over $\operatorname{BSO}(k)$ and $\mathscr{M}\left(\tilde{\gamma}^{k} \times \mathbf{Z}_{2} \widetilde{B}\right)$ is the Thom space of the unoriented vector bundle $\tilde{\gamma}^{k} \times \mathbf{z}_{2} \widetilde{B}$.

Standard arguments [12, 18.3], now involving the Thom isomorphism for local orientation systems, tell us that for $j<2 k-1$ there is an isomorphism between $\pi_{j}\left(\mathscr{M}\left(\tilde{\gamma}^{k} \times_{\mathbf{Z}_{2}} \widetilde{B}\right), \infty\right) \otimes \mathbf{Q}$ and $H_{j-k}\left(\mathrm{BSO}(k) \times \mathbf{z}_{2} \widetilde{B} ; \widehat{\mathbf{Q}}\right)$. Calculations with -1 eigenvectors show that the latter is isomorphic to

$$
\begin{aligned}
& \left(\bigoplus_{m=0}^{j-k} H_{m}(\mathrm{BO}(k) ; \mathbf{Q}) \otimes H_{j-k-m}(B ; \widehat{\mathbf{Q}})\right) \\
& \quad \oplus\left(\bigoplus_{m=0}^{j-k} H_{m}(\mathrm{BO}(k) ; \widehat{\mathbf{Q}}) \otimes H_{j-k-m}(B ; \mathbf{Q})\right) .
\end{aligned}
$$

Since $j-k<k-1$ and $H_{*}(\mathrm{BO}(k) ; \widehat{\mathbf{Q}})$ consists of sums of elements of the form $p_{I} e$ with the Euler class $e$ in dimension $k$ (Lemma 3.7), $H_{m}(\mathrm{BO}(k) ; \widehat{\mathbf{Q}})=0$ when $0 \leq m \leq j-k$. Also $H_{m}(\mathrm{BO}(k) ; \mathbf{Q})$ is isomorphic to $\Omega_{m}$ in that range [12, Chapter 18] and the result follows.

LEMMA 2.5. $H_{r}(B ; \widehat{\mathbf{Q}})$ has a basis $\left\{x_{r}, 1, \ldots, x_{r, \lambda_{r}}\right\}$ such that for each $1 \leq i \leq \lambda_{r}$ there is a singular manifold $\left(M_{i}^{r}, f_{i}\right)$ with $f_{i *}\left[M_{i}^{r}\right]=$ $x_{r, i}$.

Proof. If $\Upsilon$ is oriented this is Theorem 15.3 [4]. Otherwise by that theorem $H_{r}\left(B^{\prime}, \mathbf{Q}\right)$ has a basis $\left\{y_{1}, \ldots, y_{\mu_{r}}\right\}$ for which there are singular manifolds $\left(P_{i}^{r}, g_{i}\right)$ with $g_{i *}\left[P_{i}^{r}\right]=y_{i}$. Here $\beta: B^{\prime} \rightarrow B$ is the orientation double cover for the bundle $\Upsilon$. One can then construct a basis $\left\{\tilde{x}_{r, 1}, \ldots, \tilde{x}_{r, \lambda_{r}}\right\}$ for $H_{r}\left(B^{\prime}, \mathbf{Q}\right)^{-}$and singular manifolds $\left(M_{i}^{r}, \tilde{f}_{i}\right)$ with $\tilde{f}_{i *}\left[M_{i}^{r}\right]=\tilde{x}_{r, i}$, and let $f_{i}=\beta \tilde{f}_{i}, x_{r, i}=$ $\beta_{*} \tilde{x}_{r, i}$.

We proceed in a manner analogous to $[4$, Chapter II] to define invariants for this bordism theory. Let $x^{r, i} \in H^{r}(B ; \widehat{\mathbf{Q}})$ so that 
$\left\langle x^{r, i}, x_{r, j}\right\rangle=\delta_{i j}$. Suppose $[M, f]_{t} \in \Omega_{l, t}(\Upsilon)$. For $r$ an integer with $l \equiv r \bmod 4$ and $I$ a partition of $(l-r) / 4$ let $p_{I, r, j}[M, f]$ denote the number $\left\langle p_{I} T M \cup f^{*} x^{r, j},[M]\right\rangle$. Standard arguments like those in [4, Chapter II, §17] yield that these numbers are zero if $[M, f]_{t}=0$ and then define elements of $\operatorname{Hom}\left(\Omega_{l, t}(\Upsilon), \mathbf{Q}\right)$.

Let $0 \leq r_{1}<r_{2}<\cdots<r_{\omega} \leq l$ be those integers with $l \equiv r_{s} \bmod 4$ and $H_{r_{s}}(B ; \widehat{\mathbf{Q}}) \neq 0$. Consider triples $(s, k, K)$ where $1 \leq s \leq \omega$, $1 \leq k \leq \lambda_{r_{s}}$ and $K$ is a partition of $\left(l-r_{s}\right) / 4$. Let $R$ be the square matrix $\left(p_{I, r_{b}, i}\left[V_{J} \times M_{j}^{r_{m}}, f_{j} \pi_{2}\right]\right)$ where the rows are indexed lexicographically by triples $(b, i, I)$ as above and the columns by $(m, j, J)$. Here if $J=\left\{a_{1}, \ldots, a_{k}\right\}$ is a partition of $v$ then $V_{J}$ denotes the generator $\mathbf{C} P^{2 a_{1}} \times \cdots \times \mathbf{C} P^{2 a_{k}}$ of $\Omega_{4 v}$.

LEMMA 2.6. The matrix $R$ is nonsingular.

Proof. For $1 \leq b, m \leq \omega$ let $R_{m}^{b}$ be the submatrix of $R$ with entries $p_{*, r_{b}, *}\left[V_{*} \times M_{*}^{r_{m}}, f_{*} \pi_{2}\right]$, and then, for $1 \leq i \leq \lambda_{r_{b}}$ and $1 \leq j \leq \lambda_{r_{m}}$ let $R_{m, j}^{b, i}$ be the submatrix of $R_{m}^{b}$ with entries $p_{*, r_{b}, i}\left[V_{*} \times M_{j}^{r_{m}}, f_{j} \pi_{2}\right]$.

We first note that the matrix $R_{m}^{b}$ is zero if $b>m$, since then $r_{b}>r_{m}$ and $f_{j}^{*} x^{r_{b}, i} \in H^{r_{b}}\left(M_{j}^{r_{m}}: \mathbf{Q}\right)$ can only be zero for dimension reasons. If we show that $R_{b}^{b}$ is nonsingular for all $b$ then we will have that $R$ is nonsingular. To do this we prove that $R_{b, j}^{b, i}$ is zero if $i \neq j$ and nonsingular if $i=j$. It can be shown that

$$
p_{I, r_{b}, i}\left[V_{J} \times M_{j}^{r_{m}}, f_{j} \pi_{2}\right]=\sum_{K+L=I}\left\langle p_{K}\left(T M_{j}^{r_{m}}\right) \cup f_{j}^{*} x^{r_{b}, i},\left[M_{j}^{r_{m}}\right]\right\rangle p_{L}\left[V_{J}\right] .
$$

Also $p_{K}\left(T M_{j}^{r_{b}}\right) \cup f_{j}^{*} x_{b}^{r_{b}, i}=0$, unless $K=0$, in which case

$$
\left\langle p_{0}\left(T M_{j}^{r_{b}}\right) \cup f_{j}^{*} x^{r_{b}, i},\left[M_{j}^{r_{b}}\right]\right\rangle=\left\langle x^{r_{b}, i}, f_{j *}\left[M_{j}^{r_{b}}\right]\right\rangle=\delta_{i j} .
$$

Hence $R_{b, j}^{b, i}$ is the zero matrix if $i \neq j$, and when $i=j$ the matrix with entries $p_{I}\left[V_{J}\right]$, which by Theorem $16.8[10]$ is nonsingular.

LEMMA 2.7. If all $p_{I, r, j}[M, f]$ are zero then some multiple of $[M, f]_{t}$ is zero.

Proof. Since $R$ is nonsingular the $p_{I, r_{b}, i}$ are linearly independent and thus by dimensional considerations from Lemma 2.4 form a basis for $\operatorname{Hom}\left(\Omega_{l, t}(\Upsilon), \mathbf{Q}\right)$. From this it follows that if all the 
$p_{I, r_{b}, i}[M, f]$ are zero then $[M, f]_{t}$ is zero in $\Omega_{l, t}(\Upsilon) \otimes \mathbf{Q}$. In addition we get that the $\left[V_{J} \times M_{j}^{r_{m}}, f_{j} \pi_{2}\right]_{t}$ are linearly independent and so form a basis of $\Omega_{l, t}(\Upsilon) \otimes \mathbf{Q}$. We call such a basis formed in this way a preferred basis.

For $H$ maximal in $\mathscr{G}_{Q}, \nu_{H}$ has a classifying map $f: Q^{H} \rightarrow$ $B N_{O(d)}(H) / H$ and so our $H$ characteristic numbers are defined by $\left\langle p_{I}\left(T Q^{H}\right) \cup f^{*} x,\left[Q^{H}\right]\right\rangle$ for $x \in H^{n-d-4 s}\left(B\left(N_{\mathrm{O}(d)}(H) / H\right) ; \widehat{\mathbf{Q}}\right)$ and $I$ a partition $\left(i_{1}, \ldots, i_{m}\right)$ of $s$. These numbers are linear combinations of the $p_{I, r, j}\left[Q^{H}, f\right]$ 's.

LEMMA 2.8. If all the $H$ characteristic numbers of $Q$ are zero then some nonzero multiple of $\nu_{H}$ bounds as in Lemma 2.2.

Proof. This follows from the previous lemma where $\Upsilon$ is the canonical $\left(\mathbf{R}^{d} / H, N_{\mathrm{O}(d)}(H) / H\right)$ bundle over $B N_{\mathrm{O}(d)}(H) / H$. If $(M, f)$ bounds $(W, g)$ we may assume that $g$ is smooth and hence that $g^{*} \Upsilon$ is smooth.

When $H$ is nonmaximal $Q^{H}$ need not be an orbifold as there may be crossings at those points with local groups larger than $(H)$, (e.g. $\left.\left\langle e^{2 \pi i / p}\right\rangle \times\langle 1\rangle<\left\langle e^{2 \pi i / p}\right\rangle \times\left\langle e^{2 \pi i / p}\right\rangle\right)$ which we break apart as follows. For every $x \in Q^{H}$ choose precisely one linear chart $\left(U_{x}, G_{x}, \bar{U}_{x}, \pi_{x}\right)$ with $H<G_{x}$ and $\pi_{x}(0)=x$. Let $\left[H_{1}^{x}\right], \ldots,\left[H_{k_{x}}^{x}\right]$ be those distinct conjugacy classes of $G_{x}$ with $H_{i}^{x}$ conjugate to $H$ in $\mathrm{O}(n)$ and $H_{1}^{x}=$ $H$.

DEFINITION 2.9. $\left|Q_{H}\right|=\left\{\left(x,\left[H_{i}^{x}\right]\right): x \in Q^{H}, 1 \leq i \leq k_{x}\right\}$.

This construction is a refinement of $\widetilde{\Sigma} X$ in $[9, \S 1]$. One. can give explicit charts for $Q_{H}[6, \S 3.1 ; 9, \S 1]$. Locally $Q_{H}$ looks like $\bar{U}^{H_{i}^{x}} /\left(N_{G_{x}}\left(H_{i}^{x}\right) / K_{i}^{x}\right)$ where $K_{i}^{x}=\left\{k \in N_{G_{x}}\left(H_{i}^{x}\right): k y=y\right.$ for all $\left.y \in \bar{U}_{x}^{H_{i}^{x^{x}}}\right\}$. The overlap maps are essentially restrictions of those of $Q$.

ReMARK 2.10. (i) We can write $Q_{H}$ as $\amalg_{0 \leq j \leq r} Q_{H}^{j}$ where $Q_{H}^{j}$ is the set of elements $\left(x,\left[H_{i}^{x}\right]\right)$ of $Q_{H}$ such that $\left(K_{i}^{x}\right)$ is constant. We choose a representative $K_{j}$ of this class so that $K_{j}>H$. We also assume that $K_{0}=H$. Thus $Q_{H}^{0}=\varnothing$ if $H \notin \mathscr{G}_{Q}$. (ii) If $(H)$ is maximal in $\mathscr{G}_{Q}$, then $Q^{H}=Q_{H}=Q_{H}^{0}$. (iii) Since $\partial\left(\bar{U}_{x}^{K_{i}^{x}}\right)=$ $\partial\left(\bar{U}_{x}\right)^{K_{t}^{x}}, \partial_{o}\left(Q_{H}^{i}\right)=\left(\partial_{o} Q\right)_{H}^{i}$. 
We have that $T \bar{U}_{x \mid \bar{U}_{t}^{H^{x}}}=T \bar{U}_{x}^{H_{i}^{x}} \oplus \nu\left(\bar{U}_{x}^{H_{i}^{x}}\right)$ and the differentials of the overlap maps and of the elements of $N_{G_{x}}\left(H_{i}^{x}\right)$ factors accordingly. Define the normal orbibundle of $Q_{H}$ to be

$$
\nu_{H}=\left(\coprod_{x \in Q^{H}, 1 \leq i \leq k_{x}} \nu\left(\bar{U}_{x}^{H_{i}^{x}}\right) / N_{G_{x}}\left(H_{i}^{x}\right)\right) / \sim
$$

where $\overline{(y, v)} \sim \overline{\left(\phi(y), d \phi_{2} v\right)}$ for all appropriate overlap maps $\phi$ and $d \phi_{2}$ denotes the differential of $\phi$ in the normal direction. $\left.\nu_{H \mid}\right|_{Q_{H}^{j}}$, abbreviated $\nu_{H}^{j}$, is a $\left(\mathbf{R}^{d} / K_{j}, N_{\mathrm{O}(d)}\left(K_{j}\right) / K_{j}\right)$-orbibundle.

$T \nu_{H}$ is oriented as inherited from $Q$; however, neither $\nu_{H}$ nor $Q_{H}$ need be orientable or even locally orientable as the following two examples show.

Example 2.11. Let the dihedral group $D_{2 p}$ act on $\mathbf{R}^{\mathbf{3}}=\mathbf{C} \times \mathbf{R}$ by $\alpha\left(x_{1}, x_{2}, x_{3}\right)=\left(x_{1},-x_{2},-x_{3}\right)$ and $\beta\left(x_{1}, x_{2}, x_{3}\right)=\beta\left(z, x_{3}\right)$ $=\left(e^{2 \pi i / p} z, x_{3}\right)$. Then $Q=\mathbf{R}^{3} / D_{2 p}$ is orientable, but $Q_{\mathbf{z}_{p}}=$ $\mathbf{R}^{1} /(x \sim-x)$ is not locally orientable at $\{0\}$.

EXAmple 2.12. Let $Q^{\prime}$ be the quotient of $\mathbf{S}^{2} \times \mathbf{C}$ by the $D_{2 p}$ action: $\alpha(x, v)=(-x, \bar{v}), \beta(x, v)=\left(x, e^{2 \pi i / p} v\right) . Q^{\prime}$ is orientable. $Q_{\mathrm{Z}_{p}}^{\prime}=\mathbf{S}^{2} / D_{2 p}=R P^{2}$ is locally orientable, but not orientable.

Note that in both of these examples $N_{\mathrm{O}(2)}\left(\mathbf{Z}_{p}\right)=\mathrm{O}(2)$. However, if $N_{\mathrm{O}(d)}(H)$ is a subgroup of $\mathrm{SO}(d)$ then $\nu_{H}$ has a natural orientation and so $Q_{H}$ is oriented.

There is one exception to this induced orientation. In declaring $T \nu_{H}$ oriented as inherited from $Q$ one is assuming that the chart $\left(U_{x}, G_{x}, \bar{U}_{x}, \pi_{x}\right)$ with $H<G_{x}$ in the definition of $Q_{H}$ came from the orientation atlas of $Q$, which is always possible unless degree $H$ is $n$. In the case where we cannot have $H<G_{x}$ we give the point $x$ an orientation of -1 in $Q_{H}$. This makes sense since if $H$ has degree $n$ and is conjugate to $H^{\prime}$ in $\mathrm{O}(n)$, but not $\mathrm{SO}(n)$, one can show that $\mathbf{R}^{n} / H$ is oriented orbifold diffeomorphic, and hence cobordant, to $-\mathbf{R}^{n} / H^{\prime}$.

Definition 2.13. Let $\beta: B\left(N_{\mathrm{SO}(d)}(H) / H\right) \rightarrow B\left(N_{\mathrm{O}(d)}(H) / H\right)$ be the canonical orbit map. For $x \in H^{n-d-4 s}\left(B\left(N_{\mathrm{O}(d)}(H) / H\right) ; \widehat{\mathbf{Q}}\right)$ and $I$ a partition $\left(i_{1}, \ldots, i_{m}\right)$ of $s$ define $p_{I, x}[Q]$ to be the number

(i) $\left\langle p_{I}\left(T_{H}^{0}\right) \cup x\left(\nu_{H}^{0}\right),\left[Q_{H}^{0}\right]\right\rangle$ if $Q_{H}^{0}$ is locally oriented,

(ii) $\frac{1}{2}\left\langle p_{I}\left(T \widetilde{Q}_{H}^{0}\right) \cup \beta^{*} x\left(\tilde{\nu}_{H}^{0}\right),\left[\widetilde{Q_{H}^{0}}\right]\right\rangle$ otherwise. 
Note that the two definitions agree when $Q_{H}^{0}$ is locally orientable. The set of all such numbers, for all $x$ and $I$, are called the $H$ characteristic numbers of $Q$. Also when $H$ is maximal these are the same as the numbers defined for that case.

LemMa 2.14. If $Q=\partial_{o} W$ where $W$ is an oriented orbifold then $p_{I, x}[Q]=0$ for all $H$, and pairs $(I, x)$.

Proof. By a standard argument and Lemma $1.13 p_{I}\left(T Q_{H}^{0}\right)=$ $p_{I}\left(i^{*} T W_{H}^{0}\right)=i^{*} p_{I}\left(T W_{H}^{0}\right)$. Similarly $x\left(\nu_{H}^{0}(Q)\right)=i^{*} x\left(\nu_{H}^{0}(W)\right)$. Therefore $p_{I, x}[Q]=\left\langle i^{*}\left(p_{I}\left(T W_{H}^{0}\right) \cup x\left(\nu_{H}^{0}(W)\right)\right),\left[Q_{H}^{0}\right]\right\rangle$ which in turn is zero since $\left[Q_{H}^{0}\right]=\partial\left[W_{H}^{0}\right]$ and $i_{*} \partial=0$.

Using $Q \times I$ with the appropriate metrics at the ends it follows from the above proposition that these numbers are independent of the metric on $Q$. Also it is easy to see that the $p_{I, x}$ 's define additive homomorphisms from $\Omega_{*}$, orb to $\mathbf{Q}$. If $H, H^{\prime}$ are finite conjugate subgroups of $\mathrm{O}(n)$, one can show [6, after Lemma 3.2.7] that via the conjugation the set of $H$ characteristic numbers of $Q$ is equal to the set of $H^{\prime}$ characteristic numbers of $Q$.

THEOREM 2.15. If all the $H$ characteristic numbers of $Q$ are zero for all finite subgroups $H$ of $\mathrm{SO}(n)$ then some multiple of $Q$ orientably bounds another orbifold $W$ with $\mathscr{G}_{Q}=\mathscr{G}_{W}$.

Proof. Let $H$ be maximal in $\mathscr{G}_{Q}$. Since the $H$ characteristic numbers are all zero, by Lemmas 2.2 and 2.8 some multiple $r_{1}$ of $Q$ is cobordant to an orbifold $Q_{1}$ with $\mathscr{G}_{Q_{1}} \subset \mathscr{G}_{Q}-(H)$ via an orbifold $W_{1}$ with $\mathscr{S}_{W_{1}}=\mathscr{G}_{Q}$. Since $Q_{1} \sim_{0} r_{1} Q$, by the discussion following Lemma 2.14 all the $H^{\prime}$ characteristic numbers of $Q_{1}$ must also be zero for all $H^{\prime} \in \mathscr{G}_{Q_{1}}$.

Let $s=\operatorname{card}\left(\mathscr{G}_{Q}\right)$. Proceeding as above we get an oriented $Q_{s}$ with $Q_{s} \sim_{o} r_{s-1} Q_{s-1} \sim_{o} \cdots \sim_{o} r_{s-1} \cdots r_{1} Q$ and $\mathscr{G}_{Q_{s}}=\{e\}$. Thus $Q_{s}$ is a manifold whose Pontrjagin numbers are all zero and so $2 Q_{s}$ bounds a manifold $W_{s}$. Therefore $2 r_{s-1} r_{s-2} \cdots r_{1} Q$ orientably bounds the orbifold

$$
W=2 r_{s-1} r_{s-2} \cdots r_{2} W_{1} \cup \cdots \cup_{2 r_{s-1} Q_{s-1}} 2 W_{s-1} \cup_{2 Q_{s}} W_{s}
$$

and $\mathscr{G}_{W}=\mathscr{G}_{Q}$.

3. Analysis of the numbers. In this section we first collect a few definitions concerning real linear groups and representations. Lemmas 
3.3 and 3.4 show how the centralizer $C_{\mathrm{O}(d)}(H)$ decomposes as a product of orthogonal, unitary and symplectic groups and thus by Lemma 3.7 how $H^{*}\left(B C_{\mathrm{O}(d)}(H) ; \widehat{\mathbf{Q}}\right)$ is generated by Pontrjagin, Chern, and twisted Euler classes. This enables us to prove that $H^{s}\left(B C_{\mathrm{O}(d)}(H) ; \widehat{\mathbf{Q}}\right)$ $=0$ if $s+d$ is odd (Lemma 3.8) and then, since $H^{*}\left(B N_{\mathrm{O}(d)}(H) / H ; \widehat{\mathbf{Q}}\right)$ $<H^{*}\left(B C_{\mathrm{O}(d)}(H)\right.$; $\left.\widehat{\mathbf{Q}}\right)$ (Lemma 3.9), that every odd-dimensional orbifold rationally bounds. The $4 j+2$ case often requires further information about $N_{\mathrm{O}(d)}(H) / H$ as we indicate by considering special cases in Theorem 3.11. Finally we use a group considered in this theorem (a certain representation of $\left.S_{8}\right)$ to construct a $(4 j+2)$-dimensional oriented orbifold which does not rationally bound.

Definition 3.1. A subgroup $K$ of $\operatorname{Gl}(k, \mathbf{R})$ defines a real faithful representation of the abstract group $K . K$ is irreducible if this representation is irreducible. Suppose $K_{i}, i=1,2$, are two linear groups and $\phi_{i}: G \rightarrow \mathrm{Gl}\left(k_{i}, \mathbf{R}\right)$ are two representations of a group $G$ with $K_{i}=\varphi_{i}(G)$. Let $\beta=\left(\varphi_{1}, \varphi_{2}\right)$. By $K_{1} \oplus_{\beta} K_{2}$ we mean the linear group $\left(\varphi_{1} \oplus \varphi_{2}\right)(K)$. We will omit the subscript $\beta$ when the resulting ambiguity has no effect. $K^{m}$ means $\phi(K)^{\oplus m}$ for some faithful representation $\phi$ of $K$. If $K$ is a subgroup of $\operatorname{Gl}(k, \mathbf{R})$ and has no trivial summands then the degree of $K$ is $k$.

Definition 3.2. A real irreducible linear group is of symplectic (or $\mathbf{H}$ ) type if it has degree $4 k$ and is conjugate in $\mathrm{Gl}(4 k, \mathbf{R})$ to a subgroup of $\mathrm{Gl}(k, \mathbf{H})$. It is of complex (or $\mathbf{C}$ ) type if it has degree $2 k$ and is conjugate in $\mathrm{Gl}(2 k, \mathbf{R})$ to a subgroup of $\mathrm{Gl}(k, \mathbf{C})$ but is not of symplectic type. Otherwise it is of real (or $\mathbf{R})$ type. A real linear group is of real, complex, or symplectic type if all its irreducible components are of that type.

For $L=\mathbf{R}, \mathbf{C}, \mathbf{H}$ and $i$ any positive integer let $L(i)=\mathrm{O}(i), \mathrm{U}(i)$, $\operatorname{Sp}(i)$, and $d(L)=1,2,4$, respectively. Let $H$ be a finite subgroup of $\mathrm{O}(d)$ of degree $d$. Schur's Lemma, Theorems 1.10 and 6.7 of Chapter II, [3], imply the following two lemmas.

LEMMA 3.3. If $H$ is irreducible of type $L$ then $C_{\mathrm{O}(m d)}\left(H^{m}\right) \cong$ $L(m)$. Here we are identifying $L(1)<L(d / d(L))$, with the group of scalar matrices in $L(d / d(L))$, and thus with a subgroup of $\mathrm{O}(d)$. Note that under this identification $\mathrm{O}(1)<\mathrm{SO}(d)$ iff $d$ is even.

LEMMA 3.4. In general we can write $H$ as $H=\bigoplus_{i=1}^{r} K_{i}^{m_{i}}$ where $K_{i}$ are nonequivalent irreducible representations of $H$ in $\mathrm{O}\left(d_{i}\right)$. Then $C_{\mathrm{O}(d)}(H) \cong \times_{i=1}^{r} C_{\mathrm{O}\left(m_{i} d_{t}\right)}\left(K_{i}^{m_{i}}\right)$. 
REMARK 3.5. From the above lemmas we conclude that $C_{\mathrm{O}(d)}(H)<$ $\mathrm{SO}(d)$ if and only if $H$ has no irreducible direct summand of odd degree.

Definition 3.6. If all the irreducible components of $H$ are of even, respectively odd degree then $H$ is of even, respectively, odd type. Note that an odd type group must be of real type.

LEMMA 3.7. (i) $H^{*}(B L(m)$; Q) is a polynomial algebra with generators

(a) the symplectic Pontrjagin classes $x_{i}$ of $\operatorname{dim} 4 i, i=1, \ldots, m$, when $L=\mathbf{H}$,

(b) the Chern classes $c_{i}$ of $\operatorname{dim} 2 i, i=1, \ldots, m$, when $L=\mathbf{C}$,

(c) the Pontrjagin classes $p_{i}$ of $\operatorname{dim} 4 i, i=1, \ldots,[m / 2]$, when $L=\mathbf{R}$.

(ii) $H^{*}(\mathrm{BO}(2 m+1) ; \widehat{\mathbf{Q}})=0$ and $H^{*}(\mathrm{BO}(2 m) ; \widehat{\mathbf{Q}})$ is the graded vector space with basis $p_{i_{1}} \cdots p_{i_{1}}$ e where $1 \leq i_{k} \leq m$ for $1 \leq k \leq j$ and $e$ is the twisted Euler class of dimension $2 \mathrm{~m}$.

Proof. (i) is a standard result found in $[1,8]$. The classes in (ii) are the -1 eigenvectors of $\alpha^{*}$ where $\alpha$ is the orientation reversing involution on $\mathrm{BSO}(j)$.

LEMMA 3.8. If $H^{s}\left(B C_{\mathrm{O}(d)}(H)\right.$; $\left.\widehat{\mathbf{Q}}\right)$ is nonzero then $d+s$ is even.

Proof. Write $H$ as in Lemma 3.4. Then by that lemma

$$
H^{*}\left(B C_{\mathrm{O}(d)}(H) ; \widehat{\mathbf{Q}}\right) \cong \bigotimes_{1 \leq i \leq r} H^{*}\left(B C_{\mathrm{O}\left(m_{i} d_{i}\right)}\left(K_{i}^{m_{i}}\right) ; \widehat{\mathbf{Q}}\right) .
$$

If $H$ is of even type then by Remark 3.5 the orientation systems of the canonical bundles over the $B C_{\mathrm{O}\left(m_{l} d_{i}\right)}\left(K_{i}^{m_{l}}\right)$ 's are all trivial. Then by Lemmas 3.3, 3.4 and 3.7(i) each nonzero $H^{s}\left(B C_{\mathrm{O}\left(m_{t} d_{t}\right)}\left(K_{i}^{m_{t}}\right)\right.$; $\left.\widehat{\mathbf{Q}}\right)$ is even dimensional and so $s+d$ is even.

If $H$ is of odd type $H^{*}\left(B C_{\mathrm{O}\left(m_{i} d_{t}\right)}\left(K_{i}^{m_{i}}\right) ; \widehat{\mathbf{Q}}\right) \cong H^{*}\left(\mathrm{BO}\left(m_{i}\right) ; \widehat{\mathbf{Q}}\right)$ and we conclude [6, $\S 5.1]$ that $H^{*}\left(B C_{\mathrm{O}(d)}(H) ; \widehat{\mathbf{Q}}\right)$ is 0 if some $m_{i}$ is odd and otherwise $\mathbf{Q}\left\langle p_{I} e_{1} \cdots e_{r}\right\rangle$, the graded rational vector space with basis $p_{I} e_{1} \cdots e_{r}$, the $e_{i}$ 's denoting the Euler classes (of $\left.\operatorname{dim} m_{i}\right)$ and $p_{I}$ any appropriate product of Pontrjagin classes. Thus if $H^{s}\left(B C_{\mathrm{O}(d)}(H) ; \widehat{\mathbf{Q}}\right) \neq 0, d+s=\sum_{i=1}^{r} d_{i} m_{i}+4|I|+\sum_{i=1}^{r} m_{i}$, which is divisible by four. 
If $H$ is of neither type we can write $H \cong H_{1} \oplus H_{2}$ where $H_{1}$ is of odd type and $\mathrm{H}_{2}$ is of even type and combine the above results for our lemma.

LeMMA 3.9. Let $H$ be a finite subgroup of $\mathrm{SO}(d)$. Then

(i) The projection $\pi: N_{\mathrm{O}(d)}(H) \rightarrow N_{\mathrm{O}(d)}(H) / H$ induces an isomorphism $B \pi^{*}: H^{*}\left(B\left(N_{\mathrm{O}(d)}(H) / H\right) ; \widehat{\mathbf{Q}}\right) \rightarrow H^{*}\left(B\left(N_{\mathrm{O}(d)}(H)\right) ; \widehat{\mathbf{Q}}\right)$

(ii) The inclusion $i: C_{\mathrm{O}(d)}(H) \rightarrow N_{\mathrm{O}(d)}(H)$ induces an isomorphism of $H^{*}\left(B\left(N_{\mathrm{O}(d)}(H)\right) ; \widehat{\mathbf{Q}}\right)$ onto a subgroup of $H^{*}\left(B\left(C_{\mathrm{O}(d)}(H)\right) ; \widehat{\mathbf{Q}}\right)$.

Proof. (i) Consider the fibration

$$
B H \rightarrow B N_{\mathrm{O}(d)}(H) \rightarrow B\left(N_{\mathrm{O}(d)}(H) / H\right)
$$

of classifying spaces. Since $H$ is finite $H^{*}(B H ; \mathbf{Q})=0$. Also $H$ is orientation preserving, and so the result follows by the Serre spectral sequence.

(ii) $N_{\mathrm{O}(d)}(H) / C_{\mathrm{O}(d)}(H)$ is finite as it embeds in $\operatorname{Aut}(H)$. Thus we get the covering space $N_{\mathrm{O}(d)}(H) / C_{\mathrm{O}(d)}(H) \rightarrow B C_{\mathrm{O}(d)}(H) \stackrel{B i}{\rightarrow} B N_{\mathrm{O}(d)}(H)$ and (ii) from transfer theory.

THEOREM 3.10. If $Q$ is an odd dimensional oriented orbifold then some multiple of $Q$ bounds an oriented orbifold $W$ with $\mathscr{G}_{W}=\mathscr{G}_{Q}$.

Proof. The $H$ characteristic number $p_{I, x}[Q]$ is defined when $4|I|+$ $\operatorname{dim} x=\operatorname{dim} Q-\operatorname{deg} H$. Thus, if $\operatorname{dim} Q$ is odd, $\operatorname{dim} x+\operatorname{deg} H$ is odd, and by the previous two lemmas $x=0$. Consequently all the orbifold characteristic numbers of $Q$ are zero and our result follows from Theorem 2.15 .

We now turn to the $(4 j+2)$-dimensional case. Let $\mathscr{S O}_{f}$ be the set of all finite subgroups of $\mathrm{SO}(m)$ where $m$ is allowed to vary over all positive integers. Let $\mathscr{H}$ denote the set of elements $H$ of $\mathscr{S O}_{f}$ such that $H^{s}\left(B N_{\mathrm{O}(d)}(H) ; \widehat{\mathbf{Q}}\right)$ is nonzero implies $s+d$ is divisible by four. Here $d$ is the degree of $H$. As in Theorem 3.10 if $\operatorname{dim} Q$ is not divisible by four and $\mathscr{G}_{Q} \subset \mathscr{H}$ then some multiple of $Q$ bounds.

Theorem 3.11. Suppose $H \in \mathscr{S O}_{f}$.

(i) If $H$ is of odd type then $H \in \mathscr{H}$.

(ii) $H_{1}, H_{2} \in \mathscr{H}$ implies $H_{1} \times H_{2} \in \mathscr{H}$.

(iii) If $H$ is of complex type and conjugate to a group which is invariant under complex conjugation then $H \in \mathscr{H}$. 
(iv) If $H$ is a direct sum of symplectic type linear groups and groups of the form $K^{m}$ with $K$ irreducible, of real type and $\operatorname{deg} K$ odd or $\operatorname{deg} K^{m}$ divisible by four, then $H$ is in $\mathscr{H}$.

(v) All abelian elements of $\mathscr{S O}_{f}$ belong to $\mathscr{H}$.

(vi) $\mathscr{H}$ is a proper subset of $\mathscr{S O}_{f}$.

Proof. (i) This follows from the proof of Lemma 3.8.

(ii) Let $d_{i}=\operatorname{deg} H_{i} . \quad N_{\mathrm{O}\left(d_{1}+d_{2}\right)}\left(H_{1} \times H_{2}\right)$ has $C_{\mathrm{O}\left(d_{1}+d_{2}\right)}\left(H_{1} \times H_{2}\right)$ $\left(\cong C_{\mathrm{O}\left(d_{1}\right)}\left(H_{1}\right) \times C_{\mathrm{O}\left(d_{2}\right)}\left(H_{2}\right)\right)$ and therefore $N_{\mathrm{O}\left(d_{1}\right)}\left(H_{1}\right) \times N_{\mathrm{O}\left(d_{2}\right)}\left(H_{2}\right)$ as a finite index subgroup. By transfer $H^{*}\left(B N_{\mathrm{O}\left(d_{1}+d_{2}\right)}\left(H_{1} \times H_{2}\right)\right.$; $\left.\widehat{\mathbf{Q}}\right)$ is contained in $H^{*}\left(B N_{\mathrm{O}\left(d_{1}\right)}\left(H_{1}\right) ; \widehat{\mathbf{Q}}\right) \otimes H^{*}\left(B N_{\mathrm{O}\left(d_{2}\right)}\left(H_{2}\right) ; \widehat{\mathbf{Q}}\right)$.

(iii) Write $H$ as in Lemma 3.4 where now each $K_{j}$ is of complex type. We may also assume that this direct sum is closed under complex conjugation in $\mathrm{O}(d)$, which is conjugation by the diagonal matrix, $\alpha$, with $(1,-1, \ldots, 1,-1)$ down the diagonal. Thus $N_{\mathrm{O}(d)}(H)$ contains the semidirect product $\left(\times_{j=1}^{s} U\left(m_{j}\right)\right) \times_{\theta}\langle\alpha\rangle$ and hence $H^{*}\left(B N_{\mathrm{O}(d)}(H) ; \widehat{\mathbf{Q}}\right)<H^{*}\left(B\left(\left(\times_{j=1}^{s} U\left(m_{j}\right)\right) \times_{\theta}\langle\alpha\rangle\right) ; \widehat{\mathbf{Q}}\right)$. Since $\alpha \in \mathrm{SO}(d)$ if and only if $d$ is divisible by four, we can show that $H^{*}\left(B\left(\left(\times_{j=1}^{s} U\left(m_{j}\right)\right) \times_{\theta}\langle\alpha\rangle\right) ; \widehat{\mathbf{Q}}\right)$ is the graded vector space with basis consisting of those $c_{I_{1}} \cdots c_{I_{s}}$ with $\operatorname{dim} c_{I_{1}} \cdots c_{I_{s}} \equiv d \bmod 4$, where $c_{I_{j}}$ is any product of Chern classes in $H^{*}\left(B U\left(m_{j}\right) ; \mathbf{Q}\right)$.

(iv) Write $H$ as $K_{1} \oplus K_{2} \oplus K_{3}$ where $K_{1}$ is of symplectic type, $K_{2}$ is of real and even type, and $K_{3}$ is of real and odd type. Let $d_{i}=\operatorname{deg} K_{i}$ and $s_{i}$ be an integer such that $H^{s}\left(B C_{\mathrm{O}\left(d_{i}\right)}\left(K_{i}\right) ; \widehat{\mathbf{Q}}\right) \neq 0$. Both $d_{1}$ and $d_{2}$ are divisible by four, as are $s_{1}$ and $s_{2}$ from Lemma 3.7 (i) and Remark 3.5. By the proof of Lemma $3.8 d_{3}+s_{3}$ is also divisible by four.

(v) Every irreducible abelian group in $\mathscr{S O}_{f}$ is either of complex type and acts on $\mathbf{C}$, or $\mathbf{Z}_{2}$ and acts on $\mathbf{R}$. Hence we can write any abelian group $H$ in $\mathscr{S O}_{f}$ as $H_{1} \oplus H_{2}$ where $H_{1}$ is of complex type of degree $d_{1}$ and $H_{2}$ is a direct sum of $\phi_{j}\left(\left(\mathbf{Z}_{2}\right)^{m}\right)$ 's with $\phi_{j}$ irreducible. By taking a conjugate in $\mathrm{O}\left(d_{1}\right)$ we may assume that $H_{1}$ is closed under conjugation by the matrix $\alpha_{d_{1}}$, as in (iii) and so the matrix $\alpha_{d_{1}} \oplus \mathrm{id}_{d_{2}}$ is an element of $N_{\mathrm{O}(d)}(H)$. Also $\phi_{j}$ has degree one, so we combine (iii) and (iv) to obtain our result.

(vi) For $H \in \mathscr{S O}_{f}$ irreducible of real, even type

$$
H^{*}\left(B C_{\mathrm{O}(m d)}\left(H^{m}\right) ; \widehat{\mathbf{Q}}\right)=\mathbf{Q}\left[p_{I}\right] .
$$

If $m d$ is not divisible by four there is not enough information to 
draw the conclusion of (iv). For example, there may be no orientation reversing involution in $N_{\mathrm{O}(m d)}\left(H^{m}\right)$ to make our coefficient system nontrivial and hence kill off the Pontrjagin classes. If one had such an involution of an orientation preserving linear group $H$ with $C_{\mathrm{O}(d)}(H)=C_{\mathrm{SO}(d)}(H)$ it would have to be an outer automorphism of $H$. This tells us that a possible counterexample might be $S_{n}, n \neq 2,6$, as these groups are complete. In fact $S_{8}$ [11, character table] does have an irreducible, faithful character $\chi$ of degree seventy with $\chi(\sigma)=10$ for $\sigma$ a transposition. Furthermore all symmetric group representations have real type so this can be realized by a subgroup $H$ of $\mathrm{O}(70)$. If $A$ is a transposition in $H \operatorname{tr}(A)=10$ and $\operatorname{deg} H=70$ implies that $A$ is orientation preserving. As $H$ is generated by transpositions we conclude that $H$ is orientation preserving and hence an element of $\mathscr{S O}_{f}$. Since $H$ is complete, $H^{*}\left(B\left(N_{\mathrm{O}(70)}(H) / H\right) ; \widehat{\mathbf{Q}}\right)=H^{*}\left(B C_{\mathrm{O}(70)}(H) ; \mathbf{Q}\right)=\mathbf{Q}=\mathbf{Q}\left(p_{0}\right)$.

COROLlaRY 3.12. There is a $(4 j+2)$-dimensional oriented orbifold which does not rationally bound.

Proof. Let $V=\mathrm{D}^{70} / H$, with $H$ as in the proof of (vi) and the standard orientation on $\mathbf{D}^{70} . V$ has orbifold boundary $S=\mathbf{S}^{69} / H$. Since $S=\partial_{o} V$, Lemma 2.14 and Theorem 2.15 imply that some multiple $k$ of $S$ bounds an oriented orbifold $W$ with $\mathscr{G}_{W}=\mathscr{G}_{S}$. Also $\mathscr{G}_{S} \subset \mathscr{G}_{V}-(H)$. We form the oriented orbifold $Q^{70}=k V \cup_{k S} W$ and note that the only $H$ characteristic numbers are rational multiples of $p_{0}\left[Q^{H}\right]$, the orientation weighted number of points labeled $H$. There are $k$ such points-the origins of the $k V$ 's-and they all have the same orientation. Thus $p_{0}\left[Q^{H}\right]$ is nonzero and so by Lemma 2.14 no multiple of $Q$ bounds.

4. Generators of $\Omega_{* \text {, orb }} \otimes \mathbf{Q}$. Following the proof of Corollary 3.12 we construct a basis $P_{n}$ for $\Omega_{n \text {, orb }} \otimes \mathbf{Q}$ and show that $\Omega_{* \text {, orb }} \otimes \mathbf{Q}$ is a free commutative algebra over $\Omega_{*} \otimes \mathbf{Q}$ with generators a certain subset of the bases $P_{n}, n \geq 0$.

Let $\mathscr{K}_{n}$ be the set of conjugacy classes $(H)$ in $\mathrm{O}(n)$ of those finite subgroups $H$ of $\mathrm{SO}(n)$ with $H^{n-4 s-d}\left(B\left(N_{\mathrm{O}(d)}(H) / H\right) ; \widehat{\mathbf{Q}}\right) \neq 0$ for some $s \geq 0$, where $d=\operatorname{deg} H$. Note that $\mathscr{K}_{2 m+1}$ is empty by Lemma 3.8.

For each $(H) \in \mathscr{K}_{n}$ let $\Gamma_{H}$ denote the canonical $\left(\mathbf{R}^{d} / H\right.$, $\left.N_{\mathrm{O}(d)}(H) / H\right)$ bundle over $B\left(N_{\mathrm{O}(d)}(H) / H\right)$ and $\mathscr{G}_{H}=\mathscr{G}_{\mathbf{R}^{d} / H}$. Consider the vector space $\Omega_{n-d, t}\left(\Gamma_{H}\right) \otimes \mathbf{Q}$ and its basis $\left[V_{J} \times M_{j}^{r_{m}}, f_{j} \pi_{2}\right]_{t}$ 
indexed by $(m, j, J)$, as in $\S 2$ and the proof of Lemma 2.7. Let $\Upsilon$ denote $\left(f_{j} \pi_{2}\right)^{*} \Gamma_{H}$. Since $\mathbf{S} \Upsilon=\partial_{o} \mathbf{D} \Upsilon$ Lemma 2.14 and Theorem 2.15 imply that a nonzero multiple $k$ of $S \Upsilon$ bounds an oriented orbifold $W$ with $\mathscr{G}_{W}=\mathscr{G}_{H}-(H)$. We let $Q(H, m, j, J)=k \mathbf{D} \Upsilon \cup_{k S \Upsilon} W$ and define a linear map

$$
\Phi: S \stackrel{\text { "def" }}{=} \bigoplus_{\mathscr{K}_{n}} \Omega_{n-d, t}\left(\Gamma_{H}\right) \otimes \mathbf{Q} \rightarrow \Omega_{n, \text { orb }} \otimes \mathbf{Q}
$$

by mapping $k\left[V_{J} \times M_{j}^{r_{m}}, f_{j} \pi_{2}\right]_{t}$ in $\Omega_{n-d, t}\left(\Gamma_{H}\right) \otimes \mathbf{Q}$ to $Q(H, m, j, J)$.

Proposition 4.1. $\Phi$ is a rational vector space isomorphism.

Proof. If we view $S$ as the vector space with basis indexed by $H$ characteristic classes $p_{I, r_{b}, i}$ with $H$ ranging over $\mathscr{K}_{n}$ we see by Lemma 2.14 and Theorem 2.15 that there is an injective linear map $\lambda$ from $\Omega_{n \text {, orb }} \otimes \mathbf{Q}$ to $S$ sending an orbifold to its $H$ characteristic numbers. We note that $\left.\lambda \Phi\right|_{\Omega_{n-d, t}\left(\Gamma_{H}\right) \otimes \mathbf{Q}}$ is injective. Indeed, for $x \in$ $H^{n-d-4|I|}\left(B\left(N_{\mathrm{O}(d)}(H) / H\right) ; \widehat{\mathbf{Q}}\right)$, and $u=\sum_{i}^{k} \alpha_{i}\left[V_{J_{i}} \times M_{j_{l}}^{r_{m_{i}}}, f_{j_{l}} \pi_{2}\right] \in$ $\Omega_{n-d, t}\left(\Gamma_{H}\right) \otimes \mathbf{Q}$,

$$
p_{I, x}(\Phi(u))=\sum_{i=1}^{k} \alpha_{i}\left\langle p_{I}\left(T V_{J_{l}} \times M_{j_{i}}^{r_{m_{l}}}\right) \cup\left(f_{j_{l}} \pi_{2}\right)^{*} x,\left[V_{J_{l}} \times M_{j_{l}}^{r_{m_{l}}}\right]\right\rangle .
$$

By Lemma 2.6 if the $\alpha_{i}$ 's are not all zero there is an $x$ for which $p_{I, x}(\Phi(u)) \neq 0$. For any $(K) \in \mathscr{K}_{n} \quad \lambda \Phi$ maps the finite dimensional vector space $S_{K}=\bigoplus_{(H) \in \mathscr{G}_{K}} \Omega_{n-d, t}\left(\Gamma_{H}\right) \otimes \mathbf{Q}$ to itself. Also by the above paragraph and the fact that for $(H) \in \mathscr{G}_{K} Q(H, m, j, J)_{K}$ is the empty set when $(H) \neq(K)$ and $k\left(V_{J} \times M_{j}^{r^{m}}\right)$ when $(H)=(K)$, we see that $\lambda \Phi$ so restricted is an isomorphism of $S_{K}$. Consequently $\lambda \Phi$ and hence $\Phi$ are isomorphisms.

Let $P_{n}$ be the set of all $Q(H, m, j, J)$ where $(H) \in \mathscr{K}_{n}$ and $\left[V_{J} \times M_{j}^{r_{m}}, f_{j} \pi_{2}\right]_{t}$ is a preferred basis for $\Omega_{n-d, t}\left(\Gamma_{H}\right) \otimes \mathbf{Q}$. By the above lemma $P_{n}$ is a basis for $\Omega_{n \text {, orb }} \otimes \mathbf{Q}$.

$Q(H, m, j, J)$ is dependent on $k$ and $W$ but if we choose another pair $k^{\prime}$ and $W^{\prime}$ and let $Q^{\prime}(H, m, j, J)=k^{\prime} \mathbf{D} \Upsilon \cup_{k^{\prime} S \Upsilon} W^{\prime}$ we see that $k Q^{\prime}(H, m, j, J) \sim_{o} k^{\prime} Q(H, m, j, J) \amalg X$ where $\mathscr{G}_{X} \subset$ $\mathscr{G}_{H}-(H)$.

Let $O_{n}=\left\{Q(H, m, j, 0) \in P_{n}: H\right.$ is not a direct product (as a linear group) $\}$. 
Proposition 4.2. $\boldsymbol{\Omega}_{* \text {, orb }} \otimes \mathbf{Q}$ is a free commutative algebra over $\Omega_{*} \otimes \mathbf{Q}$ with generating set $\left\{O_{n}, n \geq 0\right\}$.

Proof. By the preceding discussion we may assume that

$$
Q(H, m, j, J)=V_{J} \times Q(H, m, j, 0) .
$$

Also $\Omega_{2 k+1, \text { orb }} \otimes \mathbf{Q}=0$, so $[Q]_{o}\left[Q^{\prime}\right]_{o}=\left[Q^{\prime}\right]_{o}[Q]_{o}$ in $\Omega_{* \text {, orb }} \otimes \mathbf{Q}$. By the proof of Theorem 3.11 (ii) $H_{*}\left(B\left(N_{\mathrm{O}\left(d+d^{\prime}\right)}\left(H \times H^{\prime}\right) /\left(H \times H^{\prime}\right)\right)\right.$; $\left.\widehat{\mathbf{Q}}\right)$ is a subgroup of $H_{*}\left(B\left(N_{\mathrm{O}(d)}(H) / H\right) ; \widehat{\mathbf{Q}}\right) \otimes H_{*}\left(B\left(N_{\mathrm{O}\left(d^{\prime}\right)}\left(H^{\prime}\right) / H^{\prime}\right) ; \widehat{\mathbf{Q}}\right)$. Then by Lemma 2.4 some multiple of $\left[M_{j}^{r_{m}}, f_{j}\right]_{t} \in \Omega_{r_{m}, t}\left(\Gamma_{H \times H^{\prime}}\right)$ lifts to $\Omega_{r_{m}, t}\left(\Gamma_{H} \times \Gamma_{H^{\prime}}\right)$ and so can be written as

$$
\sum \alpha_{i}\left[N_{j_{i}}^{n_{i}} \times P_{j_{i}^{\prime}}^{l_{i}}, \beta\left(g_{j_{i}} \times h_{j_{i}^{\prime}}\right)\right]_{t} .
$$

Here $\left[N_{j_{i}}^{n_{i}}, g_{j_{i}}\right]_{t}$ is a preferred basis element for $\Omega_{n_{i}, t}\left(\Gamma_{H}\right),\left[P_{j_{i}^{\prime}}^{l_{i}}, h_{h_{i}^{\prime}}\right]_{t}$ one for $\Omega_{l_{i}, t}\left(\Gamma_{H^{\prime}}\right)$ and

$$
\begin{aligned}
\beta: B & \left(N_{\mathrm{O}(d)}(H) / H\right) \times B\left(N_{\mathrm{O}\left(d^{\prime}\right)}\left(H^{\prime}\right) / H^{\prime}\right) \\
& \rightarrow B\left(N_{\mathrm{O}\left(d+d^{\prime}\right)}\left(H \times H^{\prime}\right) /\left(H \times H^{\prime}\right)\right)
\end{aligned}
$$

is the natural map. Hence as at the beginning of the proof we may assume that $\left[Q\left(H \times H^{\prime}, r, j, 0\right)\right]_{o}$ is equal to $\sum \alpha_{i}\left[Q\left(H, n_{i}, j_{i}, 0\right)\right]_{o}$ $\cdot\left[Q\left(H^{\prime}, l_{i}, j_{i}^{\prime}, 0\right)\right]_{o}$.

\section{REFERENCES}

[1] A. Borel and F. Hirzebruch, Characteristic classes and homogeneous spaces I, Amer. J. Math., 80 (1958), 458-538.

[2] G Bredon, Introduction to Compact Transformation Groups, Academic Press, New York, 1972.

[3] T. Bröker and T. tom Dieck, Representations of Compact Lie Groups, Springer GTM 98, Berlin, 1985.

[4] P. E. Conner and E. E. Floyd, Differentiable Periodic Maps, Springer, Berlin, 1964.

[5] M. Davis, Unpublished Lecture notes, (1988).

[6] K. Druschel, Ph.D. Thesis, the Ohio State University, (1990).

[7] A. Haeflinger, Groupoides d'holonomie et classifiants, Structure Transverse der Feuilletages, Astérisque, 116 (1984), 70-97.

[8] D. Husemoller, Fiber Bundles, McGraw-Hill, New York, 1966.

[9] T. Kawasaki, The signature theorem for V-manifolds, Topology, 17 (1978), $75-83$.

[10] C. N. Lee and A. Wasserman, Equivariant Characteristic Numbers, Springer Lecture Notes 298, Berlin, 1972, 191-216.

[11] D. Littlewood, The Theory of Group Characters and Matrix Representations of Groups, Oxford Univ. Press, 1940. 
[12] J. Milnor and J. Stasheff, Characteristic Classes, Ann. of Math. Stud., 76 (1974).

[13] I. Satake, The Gauss-Bonnet Theorem for V-manifolds, J. Math. Soc. Japan, 9 (1957), 464-476.

[14] R. E. Stong, Notes on Cobordism Theory, Princeton Math. Notes, Princeton Univ. Press, 1968.

[15] W. Thurston, The Geometry and Topology of 3-manifolds, reproduced lecture notes, Princeton University, 1978.

[16] D. Zagier, Equivariant Pontrjagin Classes and Applications to Orbit Spaces, Springer Lecture Notes 290, Berlin (1972).

Received January 13, 1992 and in revised form January 19, 1993.

St. Louis UNIVERSITY

ST. LouIs, MO 63103 



\section{CONTENTS}

K. Auinger, Free products of combinatorial strict inverse semigroups . . . . . . . . 201

T. Dana-Picard, Generic 8-dimensional algebras with mixed basis-graph . . . . . . 229

D. Dimovski, One-parameter fixed point indices . . . . . . . . . . . . . . . . . 263

K. S. Druschel, Oriented orbifold cobordism . . . . . . . . . . . . . . . . . . . 299

A. D. Helfer, Conjugate points on spacelike geodesics or pseudo-self-adjoint MorseSturm-Liouville systems . . . . . . . . . . . . . . . . . . . . . 321

J. G. Heywood and O. D. Walsh, A counter-example concerning the pressure in the Navier-Stokes equations, as $t \rightarrow 0^{+} \ldots \ldots \ldots \ldots \ldots \ldots \ldots \ldots \ldots, \ldots \ldots \ldots$

G. Masbaum and P. Vogel, 3-valent graphs and the Kauffman bracket . . . . . . 361

R. Stong, The Jones polynomial of parallels and applications to crossing number 383

Vol. 164, No. 2 


\section{PACIFIC JOURNAL OF MATHEMATICS}

Volume $164 \quad$ No. $2 \quad$ June 1994

Free products of combinatorial strict inverse semigroups

201

KARL AUINGER

Generic 8-dimensional algebras with mixed basis-graph

229

THIERRY DANA-PICARD

One-parameter fixed point indices

263

DONCO DIMOVSKI

Oriented orbifold cobordism

299

KIMBERLY SUE DRUSCHEL

Conjugate points on spacelike geodesics or pseudo-self-adjoint

Morse-Sturm-Liouville systems

ADAM D. HELFER

A counterexample concerning the pressure in the Navier-Stokes

351 equations, as $t \rightarrow 0^{+}$

JOHN GROVES HeYwOOD and OWEN WALSH

3-valent graphs and the Kauffman bracket

Gregor Masbaum and PierRe Vogel

The Jones polynomial of parallels and applications to crossing number 383

RICHARD ANDREW STONG 